\title{
The Stanley-Reisner ideals of polygons as set-theoretic complete intersections $\mathrm{s}^{1}$
}

\author{
Margherita Barile ${ }^{2}$ \\ Dipartimento di Matematica, \\ Università di Bari "Aldo Moro", Via E. Orabona 4, \\ 70125 Bari, Italy ${ }^{3}$ \\ Naoki Terai \\ Department of Mathematics, Faculty of Culture and Education, Saga \\ University, \\ Saga 840-8502, Japan
}

\begin{abstract}
We show that the Stanley-Reisner ideal of the one-dimensional simplicial complex whose diagram is an $n$-gon is always a set-theoretic complete intersection in any positive characteristic.
\end{abstract}

Keywords: Arithmetical rank, monomial ideals, set-theoretic complete intersections.

\section{Introduction and Preliminaries}

The arithmetical rank (ara) of an ideal $I$ in a commutative Noetherian ring $R$ is the minimal number $s$ of elements $a_{1}, \ldots, a_{s}$ of $R$ such that $\sqrt{I}=\sqrt{\left(a_{1}, \ldots, a_{s}\right)}$; one can express this equality by saying that $a_{1}, \ldots, a_{s}$ generate $I$ up to radical. In general height $I \leq \operatorname{ara} I$; if equality holds, $I$ is called a set-theoretic complete intersection. The ideal $I$ is called a complete intersection in the special case where $I$ is generated by height $I$ elements. Let $X$ be a non-empty finite set of indeterminates over an algebraically closed field $K$. A simplicial complex on $X$ is a set $\Delta$ of subsets of $X$ such that for all $x \in X,\{x\} \in \Delta$ and whenever $F \in \Delta$ and $G \subset F$, then $G \in \Delta$. The elements of $\Delta$ are called faces, whereas $X$ is called the vertex set of $\Delta$, and the elements of $X$ are called the vertices of $\Delta$. If $\Delta$ consists of all subsets of its vertex set, then it is called a simplex. The simplicial complex $\Delta$ can be associated with an ideal $I_{\Delta}$ of the polynomial ring $R=K[X]$, which is generated by all monomials whose support is not a face of $\Delta ; I_{\Delta}$ is called the Stanley-Reisner ideal of $\Delta$ (over $K$ ). Its minimal monomial generators are the products of the elements of the minimal non-faces of $\Delta$, and these are squarefree monomials. In fact, this construction provides a one-to-one correspondence between the simplicial complexes on $X$ and the

\footnotetext{
${ }^{1} \mathrm{MSC}$ 2000: 13A15; 13F55, 14M10.

${ }^{2}$ Partially supported by the Italian Ministry of Education, University and Research.

3 e-mail: barile@dm.uniba.it

${ }^{4}$ e-mail: terai@cc.saga-u.ac.jp
} 
squarefree monomial ideals of $K[X]$ that do not contain elements of degree one. The quotient ring $K[\Delta]=K[X] / I_{\Delta}$ is called the Stanley-Reisner ring of $\Delta$ (over $K$ ). Its Krull dimension is equal to $\max \{|F| \mid F \in \Delta\}$, and this number, lowered by one, is defined as the dimension of $\Delta$.

If $\Delta$ is 1-dimensional, we can associate with $\Delta$ a graph $G(\Delta)$ on the same vertex set: its edges are the faces of $\Delta$ having exactly two elements. In this paper, we consider, for all integers $n \geq 3$, the simplicial complex $\Delta_{n}$ for which $G\left(\Delta_{n}\right)$ is an $n$-gon. In this case $|X|=n$, say $X=\left\{x_{1}, \ldots, x_{n}\right\}$, and

$$
I_{n}=I_{\Delta_{n}}=\left(x_{i} x_{j} \mid i \text { is not adjacent to } j \text { in the cycle } 1,2, \ldots, n\right) .
$$

Moreover, height $I_{n}=n-2$. We have that $I_{3}=\left(x_{1} x_{2} x_{3}\right)$ and $I_{4}=\left(x_{1} x_{3}, x_{2} x_{4}\right)$ are complete intersections. In [1, Example 1 it is shown that $I_{5}$ can be generated up to radical by 3 elements, and in [2], Example 2, that $I_{6}$ can be generated up to radical by 4 elements. This shows that $I_{n}$ is a set-theoretic complete intersection for $n \in\{3,4,5,6\}$. In this paper we show that this property is true for all $n \geq 3$ if the characterstic of $K$ is positive. We present a recursive procedure, which, starting from $n-3$ polynomials defining $I_{n-1}$ up to radical, constructs $n-2$ polynomials defining $I_{n}$ up to radical. To this end we develop a linear-algebraic technique, similar to the one in [2] and [3], but this time we use a resultant instead of a determinant.

\section{The recursive construction}

Let $\operatorname{char} K=p$. Fix an integer $N \geq 6$. In this section we show by induction on $n$, for $5 \leq n \leq N$, that there exist $n-2$ polynomials $f_{1}, \ldots, f_{n-2}$ defining $I_{n}$ up to radical and fulfilling certain conditions on their forms, which we will state below as (I), (II), (III). As a consequence, we will deduce that $I_{n}$ is a settheoretic complete intersection for every $n \geq 5$, since we can take $N$ arbitrarily large.

We first introduce some numerical invariants. For each $n=4, \ldots, N$, fix a positive integer $r^{(n)}$ and, for each $n=5, \ldots N$, fix an integer sequence

$$
p^{r^{(n)}}=\gamma_{1}^{(n)}, \ldots, \gamma_{n-3}^{(n)},
$$

in which $\gamma_{1}^{(n)}$ is the minimum, in such a way that, for all $n=5, \ldots, N$,

$$
r^{(n-1)}>2 r^{(n)}+N,
$$

and

$$
\sum_{i=1}^{n-3} \gamma_{i}^{(n)}=p^{s^{(n)}}
$$

where

$$
s^{(n)}=r^{(n)}+N .
$$


Then set $\alpha^{(5)}=p^{r^{(5)}}, \delta^{(5)}=p^{r^{(4)}}$ and $\beta^{(5)}=1$, and pick integers $\lambda^{(5)}>\delta^{(5)}$ and $\epsilon^{(5)}>\alpha^{(5)} \delta^{(5)}$. For all $n=6, \ldots, N$, set

$$
\delta^{(n)}=p^{r^{(n-1)}},
$$

and recursively define

$$
\begin{aligned}
& \alpha^{(n)}=\alpha^{(n-1)} p^{s^{(n-1)}}, \\
& \beta^{(n)}=\beta^{(n-1)} \lambda^{(n-1)},
\end{aligned}
$$

where, for all $n=6, \ldots, N$,

$$
\lambda^{(n)}=\alpha^{(n-1)} .
$$

Finally, for all $n=6, \ldots, N$, set

$$
\epsilon^{(n)}=\alpha^{(n-1)}\left(\delta^{(n-1)}-p^{s^{(n-1)}}\right) .
$$

Note that, for all $n=5, \ldots, N$, in view of (1) and (4), we have

$$
\delta^{(n-1)}=p^{r^{(n-2)}}>p^{2 r^{(n-1)}+N}>p^{r^{(n-1)}}=\delta^{(n)} .
$$

Moreover, for all $n=6, \ldots, N$,

$$
\lambda^{(n)}=\alpha^{(n-1)}>p^{s^{(n-2)}}>p^{r^{(n-2)}}>p^{r^{(n-1)}}=\delta^{(n)},
$$

where we have used (7), (5), (3), (1) and (4). From (8), (4), (5), (3) and (1) we deduce that, for all $n=5, \ldots, N$

$$
\begin{aligned}
\epsilon^{(n)} & =\alpha^{(n-1)}\left(p^{r^{(n-2)}}-p^{s^{(n-1)}}\right) \\
& =\alpha^{(n-1)} p^{s^{(n-1)}}\left(p^{r^{(n-2)}-s^{(n-1)}}-1\right) \\
& =\alpha^{(n)}\left(p^{r^{(n-2)}-r^{(n-1)}-N}-1\right) \\
& >\alpha^{(n)}\left(p^{2^{(n-1)}+N-r^{(n-1)}-N}-1\right) \\
& =\alpha^{(n)}\left(p^{\delta^{(n)}}-1\right)>\alpha^{(n)} \delta^{(n)} .
\end{aligned}
$$

In the sequel, for the sake of simplicity, we will throughout omit the superscript $(n-1)$, so that, e.g., $\alpha$ will stand for $\alpha^{(n-1)}$. Fix an integer $n$ such that $6 \leq$ $n \leq N$. We suppose that $I_{n-1}$ can be generated, up to radical, by polynomials $g_{1}, \ldots, g_{n-3} \in K\left[x_{1}, \ldots, x_{n-1}\right]$ such that

$$
\begin{aligned}
& g_{1}=a_{1,2} x_{2}^{\lambda \beta}+\sum_{j=3}^{n-3} a_{1, j} x_{j}^{\lambda}, \\
& g_{2}=a_{2,2} x_{2}^{\beta}+\sum_{j=3}^{n-3} a_{2, j} x_{j},
\end{aligned}
$$

and, for all $i=3, \ldots, n-4$,

$$
g_{i}=\sum_{j=3}^{n-3} a_{i, j} x_{j}
$$


and, finally

$$
g_{n-3}=\sum_{j=3}^{n-3} a_{n-3, j} x_{j}^{\alpha}+x_{1} x_{n-2}^{\alpha},
$$

where, for all indices $i$ and $j, a_{i, j} \in K\left[x_{1}, \ldots, x_{n-1}\right]$. Furthermore we assume that

(I) for all $i=1, \ldots, n-4$,

$$
a_{i, i+1}=x_{n-1}^{\gamma_{i}}+b_{i}
$$

where

$$
b_{i} \in\left(x_{3}^{\delta}, \ldots, x_{n-2}^{\delta}\right) \text {; }
$$

(II) for all indices $i=2, \ldots, n-4$ and $j=3, \ldots, n-3$ such that $j \neq i+1$, we have

$$
a_{i, j} \in\left(x_{3}^{\delta}, \ldots, x_{n-2}^{\delta}\right),
$$

moreover

$$
a_{2,2} \in\left(x_{3}^{\delta}, \ldots, x_{n-2}^{\delta}\right), \quad \text { say } a_{2,2}=\sum_{j=3}^{n-2} d_{j} x_{j}^{\delta} .
$$

(III) for all indices $j=3, \ldots, n-3$,

$$
a_{n-3, j} \in I_{n-1} \cap\left(x_{3}^{\epsilon}, \ldots, x_{n-3}^{\epsilon}\right) .
$$

We will construct $n-2$ polynomials $f_{1}, \ldots, f_{n-2} \in K\left[x_{1}, \ldots, x_{n}\right]$ that have the same form as $g_{1}, \ldots, g_{n-3}$ and generate $I_{n}$ up to radical.

First we rewrite $g_{1}$ and $g_{2}$. Equality (17) follows from $(12)$, whereas $(18)$ is a consequence of 13. and 16.

$$
\begin{aligned}
& g_{1}=a_{1,2} x_{2}^{\lambda \beta}+\sum_{j=3}^{n-3} a_{1, j}^{\prime} x_{j}, \\
& g_{2}=\sum_{j=3}^{n-2} a_{2, j}^{\prime} x_{j},
\end{aligned}
$$

where, for all indices $j=3, \ldots, n-3$,

$$
a_{1, j}^{\prime}=a_{1, j} x_{j}^{\lambda-1} \in\left(x_{3}^{\delta}, \ldots, x_{n-3}^{\delta}\right),
$$

(which is true because from the definition of $\lambda^{(5)}$ and from 10 we know that $\lambda>\delta)$, and, for $j=4, \ldots, n-2$,

$$
a_{2, j}^{\prime}=a_{2, j}+d_{j} x_{2}^{\beta} x_{j}^{\delta} \in\left(x_{3}^{\delta}, \ldots, x_{n-2}^{\delta}\right),
$$


which is true by virtue of (II). Here, according to $(13)$, we have set $a_{2, n-2}=0$. Moreover, by (I),

$$
a_{2,3}^{\prime}=a_{2,3}+d_{3} x_{2}^{\beta} x_{3}^{\delta}=x_{n-1}^{\gamma_{2}}+b_{2}^{\prime}
$$

where we have set

$$
b_{2}^{\prime}=b_{2}+d_{3} x_{2}^{\beta} x_{3}^{\delta} \in\left(x_{3}^{\delta}, \ldots, x_{n-2}^{\delta}\right) .
$$

For all $i=1, \ldots, n-3$, let $\tilde{g}_{i} \in K\left[x_{1}, \ldots, x_{n-1}, y_{2}, \ldots, y_{n-2}\right]$ be defined as follows. Let

$$
\begin{aligned}
& \tilde{g}_{1}=a_{1,2} y_{2}+\sum_{j=3}^{n-3} a_{1, j}^{\prime} y_{j} \\
& \tilde{g}_{2}=\sum_{j=3}^{n-2} a_{2, j}^{\prime} y_{j} .
\end{aligned}
$$

Then, for $i=3, \ldots, n-4$, set

$$
\tilde{g}_{i}=\sum_{j=3}^{n-3} a_{i, j} y_{j}
$$

and set

$$
\tilde{g}_{n-3}=\sum_{j=3}^{n-3} a_{n-3, j} y_{j}^{\alpha}+x_{1} y_{n-2}^{\alpha} .
$$

In the sequel the apex will replace the superscript $(n)$, so that, e.g., $\alpha^{\prime}$ will stand for $\alpha^{(n)}$. We now define $f_{1}, \ldots, f_{n-3} \in K\left[x_{1}, \ldots, x_{n}\right]$ by setting

$$
f_{1}=g_{n-3}+x_{n}^{\gamma_{1}^{\prime}} x_{2}^{\alpha \lambda \beta}
$$

and, for all $i=2, \ldots, n-3$,

$$
f_{i}=g_{i-1}+x_{n}^{\gamma_{i}^{\prime}} x_{i+1}
$$

Moreover, we set

$$
\tilde{f}_{1}=\tilde{g}_{n-3}+x_{n}^{\gamma_{1}^{\prime}} y_{2}^{\alpha}
$$

and, for all $i=2, \ldots, n-3$,

$$
\tilde{f}_{i}=\tilde{g}_{i-1}+x_{n}^{\gamma_{i}^{\prime}} y_{i+1}
$$

Then, in view of $(22), 23$ and 24 , we have that $\tilde{f}_{2}, \ldots, \tilde{f}_{n-3}$ are linear polynomials in $y_{2}, \ldots, y_{n-2}$ and, by 25 ,

$$
\tilde{f}_{1}=x_{n}^{\gamma_{1}^{\prime}} y_{2}^{\alpha}+\sum_{j=3}^{n-3} a_{n-3, j} y_{j}^{\alpha}+x_{1} y_{n-2}^{\alpha}
$$


is a homogeneous polynomial in $y_{2}, \ldots, y_{n-2}$. By (27) we also have, in view of $(22)$,

$$
\tilde{f}_{2}=\sum_{j=2}^{n-2} c_{2, j} y_{j}
$$

where

$$
c_{2, j}= \begin{cases}a_{1,2} & \text { if } j=2, \\ a_{1,3}^{\prime}+x_{n}^{\gamma_{2}^{\prime}} & \text { if } j=3, \\ a_{1, j}^{\prime} & \text { if } 4 \leq j \leq n-3, \\ 0 & \text { if } j=n-2,\end{cases}
$$

and, in view of 23$]$,

$$
\tilde{f}_{3}=\sum_{j=3}^{n-2} c_{3, j} y_{j}
$$

where

$$
c_{3, j}= \begin{cases}a_{2, j}^{\prime} & \text { if } j \neq 4 \\ a_{2,4}^{\prime}+x_{n}^{\gamma_{3}^{\prime}} & \text { if } j=4 .\end{cases}
$$

Finally, for $i=4, \ldots, n-3$ we have

$$
\tilde{f}_{i}=\sum_{j=2}^{n-2} c_{i, j} y_{j},
$$

where

$$
c_{i, j}= \begin{cases}a_{i-1, j} & \text { if } j \neq i+1, n-2, \\ a_{i-1, i+1}+x_{n}^{\gamma_{i}^{\prime}} & \text { if } j=i+1, \\ 0 & \text { if } j=n-2 .\end{cases}
$$

Here we have set $a_{n-4, n-2}=0$. Let $S$ denote the resultant of $\tilde{f}_{1}, \ldots, \tilde{f}_{n-3}$ as polynomials in the indeterminates $y_{1}, \ldots, y_{n-2}$. Then, by [6], Proposition 5.4.4,

$$
S=\tilde{f}_{1}\left(\Delta_{1}, \ldots, \Delta_{n-3}\right),
$$

where $\Delta_{1}, \ldots, \Delta_{n-3}$ are such that $\operatorname{det} B=\sum_{i=1}^{n-3} \Delta_{i} T_{i}$; here $T_{1}, \ldots, T_{n-3}$ are indeterminates over $K\left[x_{1}, \ldots, x_{n}\right]$ and

$$
B=\left(\begin{array}{cccc}
c_{2,2} & \cdots & c_{2, n-3} & c_{2, n-2} \\
\vdots & & \vdots & \vdots \\
c_{n-3,2} & \cdots & c_{n-3, n-3} & c_{n-3, n-2} \\
T_{1} & \cdots & T_{n-4} & T_{n-3}
\end{array}\right)=
$$




$\left(\begin{array}{ccccccc}x_{n-1}^{\gamma_{1}}+b_{1} & a_{1,3}^{\prime}+x_{n}^{\gamma_{2}^{\prime}} & a_{1,4}^{\prime} & \cdots & a_{1, n-4}^{\prime} & a_{1, n-3}^{\prime} & 0 \\ 0 & x_{n-1}^{\gamma_{2}}+b_{2}^{\prime} & a_{2,4}^{\prime}+x_{n}^{\gamma_{3}^{\prime}} & \ldots & a_{2, n-4}^{\prime} & a_{2, n-3}^{\prime} & a_{2, n-2}^{\prime} \\ \vdots & \vdots & \ddots & \ddots & & & \vdots \\ 0 & a_{n-6,3} & \ldots & x_{n-1}^{\gamma_{n-6}}+b_{n-6} & a_{n-6, n-4}+x_{n}^{\gamma_{n-5}^{\prime}} & a_{n-6, n-3} & 0 \\ 0 & a_{n-5,3} & a_{n-5,4} & \ldots & x_{n-1}^{\gamma_{n-5}}+b_{n-5} & a_{n-5, n-3}+x_{n}^{\gamma_{n-4}^{\prime}} & 0 \\ 0 & a_{n-4,3} & a_{n-4,4} & \ldots & a_{n-4, n-4} & x_{n-1}^{\gamma_{n-4}}+b_{n-4} & x_{n}^{\gamma_{n-3}^{\prime}} \\ T_{1} & T_{2} & T_{3} & \ldots & \ldots & T_{n-4} & T_{n-3}\end{array}\right)$.

In the second equality we have applied condition (I) and definition (21). Note that, by (I) and (2),

$$
\begin{aligned}
\Delta_{1} & =\bar{\Delta}_{1}+x_{n}^{p^{s^{\prime}}-\gamma_{1}^{\prime}}, \\
\Delta_{n-3} & =\bar{\Delta}_{n-3}+x_{n-1}^{p^{s}},
\end{aligned}
$$

where $\bar{\Delta}_{1}, \bar{\Delta}_{n-3} \in\left(x_{3}^{\delta}, \ldots, x_{n-2}^{\delta}\right)$ : this follows from conditions (I) and (II), (19), 20), since $\bar{\Delta}_{1}$ and $\bar{\Delta}_{n-3}$ belong to the ideal generated by the elements $b_{1}, b_{2}^{\prime}, b_{3}, \ldots, b_{n-4}$, the elements $a_{1, j}^{\prime}$ such that $3 \leq j \leq n-3$, the elements $a_{2, j}^{\prime}$ such that $4 \leq j \leq n-2$, and the elements $a_{i, j}$ such that $3 \leq i \leq n-4$, $3 \leq j \leq n-3$, and $j \neq i+1$. Therefore

$$
x_{n} \bar{\Delta}_{1}^{\alpha} \in I_{n} \cap\left(x_{3}^{\alpha \delta}, \ldots, x_{n-2}^{\alpha \delta}\right)
$$

and

$$
x_{1} \bar{\Delta}_{n-3}^{\alpha} \in I_{n} \cap\left(x_{3}^{\alpha \delta}, \ldots, x_{n-2}^{\alpha \delta}\right) .
$$

Now, in view of 28 and 29

$$
\begin{aligned}
S & =x_{n}^{\gamma_{1}^{\prime}} \Delta_{1}^{\alpha}+\sum_{j=3}^{n-3} a_{n-3, j} \Delta_{j-1}^{\alpha}+x_{1} \Delta_{n-3}^{\alpha} \\
& =F+x_{n}^{\gamma_{1}^{\prime}(1-\alpha)+\alpha p^{s^{\prime}}}+x_{1} x_{n-1}^{\alpha p^{s}},
\end{aligned}
$$

where

$$
F=x_{n}^{\gamma_{1}^{\prime}} \bar{\Delta}_{1}^{\alpha}+\sum_{j=3}^{n-3} a_{n-3, j} \Delta_{j-1}^{\alpha}+x_{1} \bar{\Delta}_{n-3}^{\alpha} .
$$

Now, in view of (III) and (11),

$$
\sum_{j=3}^{n-3} a_{n-3, j} \Delta_{j-1}^{\alpha} \in I_{n-1} \cap\left(x_{3}^{\epsilon}, \ldots, x_{n-3}^{\epsilon}\right) \subset I_{n} \cap\left(x_{3}^{\alpha \delta}, \ldots, x_{n-2}^{\alpha \delta}\right) .
$$


From this relation, (30) and (31) we finally conclude that

$$
F \in I_{n} \cap\left(x_{3}^{\alpha \delta}, \ldots, x_{n-2}^{\alpha \delta}\right) .
$$

Set

$$
f_{n-2}=S-x_{n}^{\gamma_{1}^{\prime}(1-\alpha)+\alpha p^{s^{\prime}}}=F+x_{1} x_{n-1}^{\alpha p^{s}} .
$$

Proposition 1 If the polynomials $g_{1}, \ldots, g_{n-3}$ generate $I_{n-1}$ up to radical, then the polynomials $f_{1}, \ldots, f_{n-2}$ generate $I_{n}$ up to radical.

Proof .-We have to show that $I_{n}=\sqrt{\left(f_{1}, \ldots, f_{n-2}\right)}$. The inclusion $\supset$ is true because $f_{1}, \ldots, f_{n-2} \in I_{n}$ : the latter statement follows from the definitions of the polynomials $f_{i}$ and the fact that

$$
I_{n}=I_{n-1} K\left[x_{1}, \ldots, x_{n}\right]+\left(x_{1} x_{n-1}, x_{2} x_{n}, \ldots, x_{n-2} x_{n}\right) .
$$

We prove the inclusion $\subset$. By Hilbert's Nullstellensatz, it is sufficient to show that whenever $\mathbf{x}=\left(x_{1}, \ldots, x_{n}\right) \in K^{n}$ annihilates all $f_{i}$, then it annihilates all elements of $I_{n}$. In the rest of the proof, for the sake of simplicity, in our notation we will identify each polynomial with its value at $\mathbf{x}$. Assume that $f_{i}=0$ for all $i=1, \ldots, n-2$. We distinguish two cases. First suppose that $S=0$. Then, in view of (33) from $f_{n-2}=0$ we deduce that $x_{n}=0$. But then, in view of (26) and (27), from $f_{1}=\cdots=f_{n-3}=0$ we derive that $g_{1}=\cdots=g_{n-3}=0$. This implies that all polynomials in $I_{n-1}$ vanish at $\mathbf{x}$. Note that 32 and 34 imply

$$
F \in I_{n-1} K\left[x_{1}, \ldots, x_{n}\right]+\left(x_{n}\right) .
$$

Hence $F=0$, so that, in view of 33 , from $f_{n-2}=0$ we conclude that $x_{1} x_{n-1}=$ 0 . In view of (34), this shows that all elements of $I_{n}$ vanish at $\mathbf{x}$. Now suppose that $S \neq 0$. Then, by [10, p. 15 , from $f_{1}=\cdots=f_{n-3}=0$ we conclude that $x_{2}=\cdots=x_{n-2}=0$. But $I_{n-1} \subset\left(x_{2}, \ldots, x_{n-2}\right)$, so that all elements of $I_{n-1}$ vanish at $\mathbf{x}$. Moreover, in view of $(32)$, we have that $F=0$. Again from $f_{n-2}=0$ we deduce that $x_{1} x_{n-1}=0$. In view of (34), we conclude that all elements of $I_{n}$ vanish at $\mathbf{x}$. This completes the proof.

Proposition 1 provides the recursive step of the construction by which we intend to prove that $I_{n}$ is a set-theoretic complete intersection. In order to achieve this goal, we still have to

(a) provide three polynomials $g_{1}, g_{2}, g_{3} \in K\left[x_{1}, \ldots, x_{5}\right]$ that generate $I_{5}$ up to radical and ensure that they fulfil conditions (I), (II) and (III), and

(b) show that the polynomials $f_{1}, \ldots f_{n-2}$ fulfil conditions (I), (II) and (III), as well.

Task (a) is accomplished if we take $\gamma_{1}=\gamma_{1}^{(5)}, \gamma_{2}=\gamma_{2}^{(5)}, \delta=\delta^{(5)}$ and $\alpha=\alpha^{(5)}$, and we consider the polynomials

$$
\begin{aligned}
& g_{1}=x_{5}^{\gamma_{1}} x_{2}+x_{1} x_{3}^{\lambda}, \\
& g_{2}=x_{4}^{\lambda} x_{2}+x_{5}^{\gamma_{2}} x_{3}, \\
& g_{3}=x_{1} x_{4}^{\alpha} .
\end{aligned}
$$


These polynomials arise from a modification of those presented in [1, Example 1. The proof can be easily performed by applying Hilbert's Nullstellensatz and considering the cases where $x_{1}=0$ and $x_{4}=0$ separately. Since $a_{1,2}=x_{5}^{\gamma_{1}}$, $a_{2,3}=x_{5}^{\gamma_{2}}$, condition (I) holds; furthermore, since $a_{2,2}=x_{4}^{\lambda}$, condition (II) holds; finally, since $a_{3,3}=0$, condition (III) holds, too. We now handle (b). To this end, we first need to write $f_{1}, \ldots, f_{n-2}$ in a suitable way.

By (15) and (26), we have

$$
f_{1}=\tilde{a}_{i, 2} x_{2}^{\alpha \lambda \beta}+\sum_{j=3}^{n-2} \tilde{a}_{1, j} x_{j}^{\alpha},
$$

where

$$
\tilde{a}_{1, j}= \begin{cases}x_{n}^{\gamma_{1}^{\prime}} & \text { if } j=2 \\ a_{n-3, j} & \text { if } 3 \leq j \leq n-3, \\ x_{1} & \text { if } j=n-2 .\end{cases}
$$

Moreover, by (12) and (27),

$$
f_{2}=\tilde{a}_{2,2} x_{2}^{\lambda \beta}+\sum_{j=3}^{n-2} \tilde{a}_{2, j} x_{j},
$$

where, by (I),

$$
\tilde{a}_{2, j}= \begin{cases}a_{1,2}=x_{n-1}^{\gamma_{1}}+b_{1} & \text { if } j=2, \\ x_{n}^{\gamma_{2}}+a_{1,3} x_{3}^{\lambda-1} & \text { if } j=3, \\ a_{1, j} x_{j}^{\lambda-1} & \text { if } 4 \leq j \leq n-3, \\ 0 & \text { if } j=n-2,\end{cases}
$$

and, by (27), (18), 20) and (21),

$$
f_{3}=\sum_{j=3}^{n-2} \tilde{a}_{3, j} x_{j},
$$

where

$$
\tilde{a}_{3, j}= \begin{cases}a_{2, j}+d_{j} x_{2}^{\beta} x_{j}^{\delta} & \text { if } j \neq 4, n-2 \\ x_{n}^{\gamma_{3}^{\prime}}+a_{2,4}+d_{4} x_{2}^{\beta} x_{4}^{\delta} & \text { if } j=4 \\ d_{n-2} x_{2}^{\beta} x_{n-2}^{\delta} & \text { if } j=n-2 .\end{cases}
$$

For $i=4, \ldots, n-3$, we further have

$$
f_{i}=\sum_{j=3}^{n-2} \tilde{a}_{i, j} x_{j}
$$

where, by (14), and 27),

$$
\tilde{a}_{i, j}= \begin{cases}a_{i-1, j} & \text { if } j \neq i+1, n-2, \\ x_{n}^{\gamma_{i}}+a_{i-1, i+1} & \text { if } j=i+1, \\ 0 & \text { if } j=n-2, i \neq n-3 .\end{cases}
$$


Here, according to 14 , we have set $a_{n-4, n-2}=0$, so that $\tilde{a}_{n-3, n-2}=x_{n}^{\gamma_{n-3}^{\prime}}$. Finally, by (32) and (33) we have that

$$
f_{n-2}=\sum_{j=3}^{n-2} a_{n-2, j} x_{j}^{\alpha p^{s}}+x_{1} x_{n-1}^{\alpha p^{s}},
$$

where, for all $j=3, \ldots, n-2$,

$$
a_{n-2, j} \in I_{n} \cap\left(x_{3}^{\epsilon^{\prime}}, \ldots, x_{n-2}^{\epsilon^{\prime}}\right),
$$

and, according to (8), $\epsilon^{\prime}=\alpha \delta-\alpha p^{s}$. Comparing (12) with (36), (13) with (38), (14) with (40) and (42), (15) with (44), we see that $f_{1}, \ldots, f_{n-2}$ are polynomials of the same form as $g_{1}, \ldots, g_{n-3}$ with $n-2$ instead of $n-3, \lambda^{\prime}=\alpha$ instead of $\lambda$ (see (7)), $\beta^{\prime}=\lambda \beta$ instead of $\beta$ (see (6) ) and $\alpha^{\prime}=\alpha p^{s}$ instead of $\alpha$ (see (5p). We show that conditions (I), (II) and (III) are fulfilled by $f_{1}, \ldots, f_{n-2}$ with respect to these new data. From (9) we know that $\delta>\delta^{\prime}$. Hence, in view of (II), for $i=4, \ldots, n-4$, we have

$$
a_{i-1, i+1} \in\left(x_{3}^{\delta}, \ldots, x_{n-2}^{\delta}\right) \subset\left(x_{3}^{\delta^{\prime}}, \ldots, x_{n-1}^{\delta^{\prime}}\right) .
$$

Therefore, in view of (43), the coefficients $\tilde{a}_{i, i+1}$, for $i=4, \ldots, n-4$, fulfil condition (I) with $n$ instead of $n-1, \tilde{b}_{i}=a_{i-1, i+1}$ instead of $b_{i}, \gamma_{i}^{\prime}$ instead of $\gamma_{i}$ and $\delta^{\prime}$ instead of $\delta$. This is also true for $i=n-3$, with $\tilde{b}_{n-3}=0$, since $\tilde{a}_{n-3, n-2}=x_{n}^{\gamma_{n-3}^{\prime}}$, and for $i=1$, with $\tilde{b}_{1}=0$, since by $\sqrt{37} \tilde{a}_{1,2}=x_{n}^{\gamma_{1}^{\prime}}$. It is also true for $i=2$, with $\tilde{b}_{2}=a_{1,3} x_{3}^{\lambda-1}$, since, by virtue of $390, \tilde{a}_{2,3}=x_{n}^{\gamma_{2}^{\prime}}+a_{1,3} x_{3}^{\lambda-1}$ and, by 10 and $(9), \lambda-1>\delta^{\prime}$. From 41) we have $\tilde{a}_{3,4}=x_{n}^{\gamma_{3}^{\prime}}+a_{2,4}+d_{4} x_{2}^{\beta} x_{4}^{\delta}$, where $a_{2,4} \in\left(x_{3}^{\delta}, \ldots, x_{n-2}^{\delta}\right)$ : this follows from (II) if $n>6$, on the other hand, for $n=6$, we have that $a_{2,4}=a_{n-4, n-2}=0$. Since, by $(9), \delta>\delta^{\prime}$, it follows that condition (I) is also true for $i=3$ with $\tilde{b}_{3}=a_{2,4}+d_{4} x_{2}^{\beta} x_{4}^{\delta}$. This establishes condition (I). Next we show that condition (II) holds for the coefficients $\tilde{a}_{i, j}$ with $\delta^{\prime}$ instead of $\delta$. From (43) and (II) we see that

$$
\tilde{a}_{i, j}=a_{i-1, j} \in\left(x_{3}^{\delta}, \ldots, x_{n-2}^{\delta}\right) \subset\left(x_{3}^{\delta^{\prime}}, \ldots, x_{n-1}^{\delta^{\prime}}\right)
$$

for all $i=4, \ldots, n-3$ and $j=3, \ldots, n-3$ such that $j \neq i$ and $j \neq i+1$. This also holds for $i=4, \ldots, n-4, j=n-2$, since $\tilde{a}_{i, n-2}=0$. From (II) and (41), we have

$$
\tilde{a}_{3, j}=a_{2, j}+d_{j} x_{2}^{\beta} x_{j}^{\delta} \in\left(x_{3}^{\delta}, \ldots, x_{n-2}^{\delta}\right) \subset\left(x_{3}^{\delta^{\prime}}, \ldots, x_{n-1}^{\delta^{\prime}}\right)
$$

for $j=5, \ldots, n-2$, where we have set $a_{2, n-2}=0$. Moreover, since $\lambda>\delta^{\prime}$, from (39) we derive that

$$
\tilde{a}_{2, j}=a_{1, j} x_{j}^{\lambda-1} \in\left(x_{4}^{\lambda-1}, \ldots, x_{n-3}^{\lambda-1}\right) \subset\left(x_{3}^{\delta^{\prime}}, \ldots, x_{n-1}^{\delta^{\prime}}\right)
$$

for all $j=4, \ldots, n-3$, whereas $\tilde{a}_{2, n-2}=0$. This establishes condition (II) for $i \neq j$. Now, from (I) and 43) we deduce that, for all $i=4, \ldots, n-3$,

$$
\begin{aligned}
\tilde{a}_{i, i} & =a_{i-1, i}=x_{n-1}^{\gamma_{i-1}}+b_{i-1} \\
& \in\left(x_{3}^{\delta}, \ldots, x_{n-2}^{\delta}, x_{n-1}^{\gamma_{i-1}}\right) \subset\left(x_{3}^{\delta^{\prime}}, \ldots, x_{n-2}^{\delta^{\prime}}, x_{n-1}^{\delta^{\prime}}\right),
\end{aligned}
$$


where we have used the fact that $\gamma_{i-1} \geq \gamma_{1}=\delta^{\prime}$. Similarly, from (I) and (39) we deduce that

$$
\tilde{a}_{2,2}=x_{n-1}^{\gamma_{1}}+b_{1} \in\left(x_{3}^{\delta^{\prime}}, \ldots, x_{n-2}^{\delta^{\prime}}, x_{n-1}^{\delta^{\prime}}\right),
$$

and from (I) and (41), since $\gamma_{2} \geq \gamma_{1}$,

$$
\tilde{a}_{3,3}=a_{2,3}+d_{3} x_{2}^{\beta} x_{3}^{\delta}=x_{n-1}^{\gamma_{2}}+d_{3} x_{2}^{\beta} x_{3}^{\delta}+b_{2} \in\left(x_{3}^{\delta^{\prime}}, \ldots, x_{n-2}^{\delta^{\prime}}, x_{n-1}^{\delta^{\prime}}\right) .
$$

This establishes condition (II). Finally, note that 450 implies that the coefficients $a_{n-2, j}$ fulfil condition (III) with $\epsilon^{\prime}$ instead of $\epsilon$. We have just proven the following result.

Theorem 1 Suppose that char $K=p>0$. Then, for all $n \geq 5$, the ideal $I_{n}$ of $K\left[x_{1}, \ldots, x_{n}\right]$ is a set-theoretic complete intersection.

\section{Some consequences}

The simplicial complexes $\Delta_{n}$ occur in some classification theorems, together with the simplicial complexes $\Lambda_{n}$ for which $G\left(\Lambda_{n}\right)$ is the straight path $x_{1}, \ldots, x_{n}$. From [8, Theorem 5.1, we know that the Stanley-Reisner ring $K[\Delta]$ of a 1dimensional simplicial complex $\Delta$ is Gorenstein (i.e., $\Delta$ is a Gorenstein complex over $K$ ) if and only if $\Delta=\Delta_{n}$ for some $n \geq 3$, or $\Delta=\Lambda_{2}$, or $\Delta=\Lambda_{3}$. The first case is the one where the $a$-invariant of $K[\Delta]$ is zero. The Stanley-Reisner ideal $I_{\Lambda_{2}}$ is the zero ideal of $K\left[x_{1}, x_{2}\right]$, and $I_{\Lambda_{3}}=\left(x_{1} x_{3}\right) \subset K\left[x_{1}, x_{2}, x_{3}\right]$ : both ideals are principal. As a consequence of Theorem 1 we thus have:

Corollary 1 Suppose that char $K>0$. If $\Delta$ is a 1-dimensional Gorenstein complex over $K$, then $I_{\Delta}$ is a set-theoretic complete intersection.

More in general, $I_{\Lambda_{n}}$ is a set-theoretic complete intersection: this can be derived, e.g., from [3, Corollary 2, by a trivial inductive argument. In 9] Terai and Yoshida call $\Delta$ a locally complete intersection complex if, for the link of every vertex, the Stanley-Reisner ideal is a complete intersection (recall that the link of a vertex $x$ is the subcomplex induced on the set of vertices distinct from $x$ that lie in the same face as $x$ ). In 9 , Theorem 1.15, they show that a non-empty simplicial complex $\Delta$ is a locally complete intersection if and only if $G(\Delta)$ is a finite disjoint union of "polygons", "straight paths", "points" and complete intersection complexes of dimension at least 2, and that under this assumption, whenever $\operatorname{dim} \Delta=1, \Delta$ is Cohen-Macaulay over $K$ if and only if $G(\Delta)$ is connected. It is well known that a complete intersection is Cohen-Macaulay. On the other hand, if it is disconnected, it is well known that depth $K[\Delta]=1$. As a consequence of the Auslander-Buchsbaum formula (see, e.g., 4, Theorem 1.3.3), we thus have projdim $K[\Delta]=n-1$, where we recall that $n=|X|$. Moreover, $\operatorname{projdim} K[\Delta] \leq$ ara $I_{\Delta}$ by [7], and ara $I_{\Delta} \leq n-1$ by [5], Theorem 2 . In view of Theorem 1 we thus obtain: 
Corollary 2 Suppose that char $K>0$. If $\Delta$ is a Cohen-Macaulay locally complete intersection complex over $K$, then $I_{\Delta}$ is a set-theoretic complete intersection. More in general, if $\Delta$ is a locally complete intersection complex over $K$, then

$$
\operatorname{projdim} K[\Delta]=\operatorname{ara} I_{\Delta}=n-1 .
$$

\section{References}

[1] Barile, M., A generalization of a lemma by Schmitt and Vogel. Preprint (2006). To appear in: Tokyo J. Math.

[2] Barile, M., Arithmetical ranks of Stanley-Reisner ideals via linear algebra, Comm. Algebra 36, (2008), 4540-4556.

[3] Barile, M., Terai, N., Arithmetical ranks of Stanley-Reisner ideals of simplicial complexes with a cone. Preprint (2008). arXiv:0809.2194. To appear in: Comm. Algebra.

[4] Bruns, W., Herzog, J. Cohen-Macaulay rings, 2nd ed., Cambridge University Press, Cambridge, 1998.

[5] Eisenbud, D. and Evans, E.G., Every algebraic set in $n$-space is the intersection of $n$ hypersurfaces, Inventiones Math. 19, (1973), 107-112.

[6] Jouanolou, J., Le formalisme du résultant, Adv. Math. 80, (1991), pp. 117263.

[7] Lyubeznik, G., On the local cohomology modules $H_{\mathcal{A}}^{i}(R)$ for ideals $\mathcal{A}$ generated by monomials in an $R$-sequence. In: Complete Intersections, Lectures given at the $1^{\text {st }} 1983$ Session of the Centro Internazionale Matematico Estivo (C.I.M.E.), Acireale, Italy, June 13-21, 1983; Greco, S., Strano, R., Eds.; Springer: Berlin Heidelberg, 1984, 214-220.

[8] Stanley, R. P., Combinatorics and Commutative Algebra, 2nd ed., Springer, New York, 2004.

[9] Terai, N., Yoshida, K.-I., Locally complete intersection Stanley-Reisner ideals. Preprint (2009). arXiv:0901.3899.

[10] Van Der Waerden, B.L., Modern Algebra, vol. II, Frederick Ungar, New York, 1950. 